\title{
Identification of anti-inflammatory
} compounds from Zhongjing formulae by knowledge mining and high-content screening in a zebrafish model of inflammatory bowel diseases

\author{
Yunru Yu ${ }^{1 \dagger}$, Jing Chen ${ }^{1+}$, Xiaohui Zhang ${ }^{1}$, Yingchao Wang ${ }^{1}$, Shufang Wang ${ }^{1}$, Lu Zhao ${ }^{1 *}$ and Yi Wang ${ }^{1,2,3^{*}}$ (D)
}

\begin{abstract}
Background: Inflammatory bowel diseases (IBD) are chronic relapsing intestinal inflammations with increasing global incidence, and new drug development remains in urgent demand for IBD management. To identify effective traditional Chinese medicine (TCM) formulae and compounds in IBD treatment, we innovatively combined the techniques of knowledge mining, high-content screening and high-resolution mass spectrometry, to conduct a systematic screening in Zhongjing formulae, which is a large collection of TCM prescriptions with most abundant clinical evidences.
\end{abstract}

Methods: Using Word2vec-based text learning, the correlations between 248 Zhongjing formulae and IBD typical symptoms were analyzed. Next, from the top three formulae with predicted relationship with IBD, TCM fractions were prepared and screened on a transgenic zebrafish IBD model for their therapeutic effects. Subsequently, the chemical compositions of the fraction hits were analyzed by mass spectrometry, and the major compounds were further studied for their anti-IBD effects and potential mechanisms.

Results: Through knowledge mining, Peach Blossom Decoction, Pulsatilla Decoction, and Gegen Qinlian Decoction were predicted to be the three Zhongjing formulae mostly related to symptoms typical of IBD. Seventy-four fractions were prepared from the three formulae and screened in TNBS-induced zebrafish IBD model by high-content analysis, with the inhibition on the intestinal neutrophil accumulation and ROS level quantified as the screening criteria. Six herbal fractions showed significant effects on both pathological processes, which were subsequently analyzed by mass spectrometry to determine their chemical composition. Based on the major compounds identified by mass spectrometry, a second-round screen was conducted and six compounds (palmatine, daidzin, oroxyloside, chlorogenic acid, baicalin, aesculin) showed strong inhibitory effects on the intestinal inflammation phenotypes. The expression of multiple inflammatory factors, including $i / 1 \beta, c / c \times 8 a, m m p$ and $t$ nfa, were increased in TNBS-treated fish, which were variously inhibited by the compounds, with aesculin showing the most potent effects. Moreover, aesculin and daidzin also upregulated e-cadherin's expression.

\footnotetext{
*Correspondence: Izhao@zju.edu.cn; zjuwangyi@zju.edu.cn

${ }^{\dagger}$ Yunru Yu and Jing Chen contributed equally to this work

1 Pharmaceutical Informatics Institute, College of Pharmaceutical

Sciences, Zhejiang University, Hangzhou 310058, China

Full list of author information is available at the end of the article
}

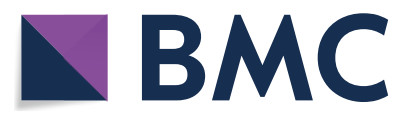

(c) The Author(s) 2021. This article is licensed under a Creative Commons Attribution 4.0 International License, which permits use, sharing, adaptation, distribution and reproduction in any medium or format, as long as you give appropriate credit to the original author(s) and the source, provide a link to the Creative Commons licence, and indicate if changes were made. The images or other third party material in this article are included in the article's Creative Commons licence, unless indicated otherwise in a credit line to the material. If material is not included in the article's Creative Commons licence and your intended use is not permitted by statutory regulation or exceeds the permitted use, you will need to obtain permission directly from the copyright holder. To view a copy of this licence, visit http://creativeco mmons.org/licenses/by/4.0/. The Creative Commons Public Domain Dedication waiver (http://creativecommons.org/publicdomain/ zero/1.0/) applies to the data made available in this article, unless otherwise stated in a credit line to the data. 
Conclusion: Taken together, we demonstrated the regulatory effects of several TCM formulae and their active compounds in the treatment of IBD, through a highly efficient research strategy, which can be applied in the discovery of effective TCM formulae and components in other diseases.

Keywords: Inflammatory bowel diseases, Zhongjing formulae, Knowledge mining, High-content screening, Zebrafish, Multimodal identification

\section{Highlights}

- TCM formulae most related to IBD was identified by knowledge mining.

- Zebrafish-based high-content analysis was used to find effective TCM components in IBD.

- Aesculin and daidzin showed both anti-inflammation and epithelia protective effects.

\section{Introduction}

Inflammatory bowel diseases (IBDs), which include Crohn's disease and ulcerative colitis, are chronic relapsing gastrointestinal tract disorders characterized by diarrhea, abdominal cramping, fatigue, weight loss, and increased risk of developing colorectal cancers [1]. Despite previously considered as a disease of westernized countries, epidemiological studies in recent years identified rapidly accelerating incidences of IBDs in newly industrialized regions in Asia, Africa and South America, which make it a global health challenge [2]. The initiation and progression of IBDs are believed to be caused by the interactive results of multiple contributors, including environmental factors, genetics factors, infections and immune dysfunction. As a result, the clinical management of IBDs is challenging [3]. Aside from traditional treatment regimens such as aminosalicylates and corticosteroids, biological therapies have been actively explored in the past decade, such as monoclonal antibodies (mAbs) to tumor necrosis factor- $\alpha$ (TNF- $\alpha$ ), interleukin (IL)-12 and IL-23. However, considering the complicate disease mechanism of IBDs, exploring new treatment options is still in high demand.

Traditional Chinese Medicine (TCM) has been used for millennia in China and many other Asian countries. However, despite numerous evidences were accumulated from clinical practices, most active compounds and targets of TCM remain ill-defined due to its complex chemical compositions, which severely hindered its interpretation by modern medicine. On the other hand, those knowledge recorded in classic TCM literatures may be overlooked. Because of the large amount and language obstacle, it is often difficult for nowadays clinicians or researchers to efficiently retrieve information from traditional TCM literatures, which were mostly written in ancient Chinese. Nevertheless, many information in these documents can be very useful. For example, the low heat resistance of the Nobel prize-winning discovery artemisinin was firstly inspired by an ancient TCM handbook [4]. IBD has been treated by TCM for thousands of years, although under the name of diverse clinical symptoms which are now included in the disease scope [5]. Possible mechanisms related to herbal medicines in IBD include maintenance of intestinal epithelial integrity, regulation of macrophage activation, modulation of immune response, and inhibition of TNF- $\alpha$ activity [6]. For example, curcumin alleviated the histopathologic changes in a trinitrobenzene sulfonic acid (TNBS)-induced mice IBD model, with suppressed $\mathrm{CD} 4^{+}$T-cell infiltration and NF- $k B$ activation [7]. Nevertheless, the effects and mechanism of many botanical drugs in IBD are still not examined.

Aiming at the aforementioned challenges of TCM drug discovery, we combined the methods of computerized knowledge mining, high-content analysis, and high-resolution mass spectrometry (HRMS) in the current study. Firstly, systematic knowledge discovery of Zhongjing formulae, which was originally compiled by Zhang Zhongjing of Eastern Han Dynasty and regarded as the "progenitor of all TCM formulae", was conducted using computerized text mining to determine the relationship between different prescriptions and clinical indications. Subsequently, high-content analysis was performed using a zebrafish IBD model, to screen for the effects of Zhongjing formulae in IBDs, the pipeline of which was further coupled with HPLC-MS, to identify active chemical compounds. The vertebrate animal model zebrafish is an ideal screening tool for high-content analysis, which has many advantages such as small body size, high fertility, rapid development, larvae transparency and low breeding cost. Moreover, zebrafish shares high conservation with mammals in most aspects, including genes regulating inflammatory signalings. TNBS is known to induce immunogenic reactions in the intestines of mice [8], and has also been used to establish IBD model in zebrafish $[9,10]$. Since neutrophils accumulation in the epithelia exerted critical roles in modulating intestinal mucosal immune responses in IBD [11], and reactive oxygen species (ROS) is an essential mediator of 
mucosal injury [12], the intestinal accumulation of neutrophils and ROS were used as markers to evaluate the disease severity of IBD and the therapeutic effects in the fish model. Through the combination of modern scientific techniques, here we reported the identification of multiple active substances in the treatment of IBDs from ancient TCM literatures.

\section{Materials and methods}

\section{Animal care ethics}

All zebrafish experiments were conducted according to the guidelines of Animal Ethics Committee of the Laboratory Animal Center, Zhejiang University.

\section{Zebrafish husbandry}

$\operatorname{Tg}$ (Lyz:DsRED2) transgenic fish [13] was obtained from the Laboratory Animal Center of Zhejiang University. Zebrafish were maintained following standard protocols [14]. E3 medium $(0.29 \mathrm{~g} / \mathrm{l} \mathrm{NaCl}, 0.013 \mathrm{~g} / \mathrm{l} \mathrm{KCl}, 0.048 \mathrm{~g} / \mathrm{l}$ $\mathrm{CaCl}_{2} \cdot 2 \mathrm{H}_{2} \mathrm{O}, 0.082 \mathrm{~g} / \mathrm{l} \mathrm{MgCl}_{2} \cdot 6 \mathrm{H}_{2} \mathrm{O}, \mathrm{pH}$ 7.2) was used as the embryo medium. Embryos were obtained through natural spawning.

\section{Chemicals and reagents}

TNBS (MB5547), 5-ASA (MB7539), and DCFH-DA (MA0219) were purchased from Dalian Meilun Biotechnology Company, China. 1-phenyl 2-thiourea (PTU) (P7629) and Ethyl 3-aminobenzoate methanesulfonate (Tricaine, E10521) were purchased from Sigma-Aldrich company of USA. Puerarin, 3'-methoxypuerarin, daidzin, oroxindin, glycyrrhizic acid and esculin were purchased from Shanghai Yuanye Bio-Technology Co., Ltd (Shanghai, China). Palmatine chloride, chlorogenic acid, oroxyloside, fraxin and phellodendrine were purchased from Shanghai Winherb Medical Technology Co., Ltd (Shanghai, China). Berberine chloride was obtained from Dalian Meilun BiotechnologyCo., Ltd (Dalian, China). Baicalin was obtained from Shanghai Aladdin Bio-Chem Technology Co., Ltd (Shanghai, China). The purities of all compounds were no less than 98\%. HPLC-grade acetonitrile, methanol and formic acid were from Merk (Darmstadt, Germany). Deionized water was prepared with an Elga PURELAB flex system (ELGA LabWater, UK).

\section{Knowledge mining}

Forty ancient TCM books derived from or closely related to Zhongjing formulae were collected (Additional file 1: Table S1). A literature search was conducted on China National Knowledge Infrastructure and NCBI Pubmed, using "Shanghan", "Zhongjing", or the names of the 248 Zhongjing formulae as keywords, in the time window between 1999/01/01 and 2018/12/31. All articles were exported as the Refworks format. The word frequencies from the text of TCM books, or the titles and abstracts of articles were counted using Python (3.6). The lists of disease-related words and their frequencies were extracted, and the top 100 common words were visualized through word cloud generator in Python. Chinese phrases dictionaries were obtained from free online resources and imported into the program to automatically distinguish different types of phrases, e.g. herbs, formulae, syndromes, and diseases.

Text training was performed using Skip-gram models from Word2vec $[15,16]$. Model files storing the words and their vectors were obtained as the output, through which the semantic distances between input and output words were calculated using cosine similarity. After descending the dimensionality, synonyms clustering can be visualized to assess the similarity among words. The resulted words vectors were visualized by $\mathrm{t}$-distributed stochastic neighbor embedding. The training was conducted by Python with following parameters: size, 100; seed, 0 ; workers, 7 . The set of word vectors was visualized by $t$-distributed stochastic neighbor embedding for dimensionality reduction.

\section{Fractions preparation and LC-MS analysis}

The fractions were prepared as previously reported [17]. The fractions were dissolved in DMSO to $50 \mathrm{mg} /$ $\mathrm{ml}$ for the stock solution for high content screening, and then diluted by water for LC-MS analysis. All the samples were centrifuged at 10,000 rpm for $20 \mathrm{~min}$, and the supernatants were subject to LC-MS analysis. Acquity UPLC system (Waters, Milford, MA, USA) coupled with Triple TOF 5600 plus MS (AB SCIEX, Framingham, MA, USA) was employed for chemical identification. Chromatographic separation was carried out on Waters ACQUITY UPLC HSS T3 $(100 \mathrm{~mm} \times 2.1 \mathrm{~mm}$ i.d. $1.8 \mu \mathrm{m})$ at $40{ }^{\circ} \mathrm{C}$ with mobile phase A $(0.1 \%$ formic acid-water) and mobile phase $\mathrm{B}$ (acetonitrile). The flow rate was $0.25 \mathrm{ml} / \mathrm{min}$. Mass spectrometry analysis was performed in both positive and negative modes under following parameters: scan range, m/z 100-2000; ion source GS1, $50 \mathrm{psi}$; ion source GS2, 50 psi; curation gas (CUR), $35 \mathrm{psi}$; temperature, $600^{\circ} \mathrm{C}$ for $\mathrm{ESI}^{+}$and $550^{\circ} \mathrm{C}$ for $\mathrm{ESI}^{-}$; ionspray voltage (IS), $-4.5 \mathrm{kV}$ for ESI ${ }^{-}$and $5.0 \mathrm{kV}$ for ESI ${ }^{+}$.

\section{Zebrafish IBD modeling and drug treatment}

For the construction of IBD model, 3 day post fertilization (dpf) zebrafish embryos were incubated in embryo medium supplemented with $75 \mu \mathrm{g} / \mathrm{ml}$ TNBS for 2 days, and $0.1 \%$ DMSO was used as a vehicle control. For drug protection, 2dpf embryos were incubated in 5-ASA $(200 \mu \mathrm{g} / \mathrm{ml})$, TCM fractions $(50 \mu \mathrm{g} / \mathrm{ml})$, or compounds $(200 \mu \mathrm{M})$ for $24 \mathrm{~h}$, and then replaced with TNBS treatment. In order to determine the concentration of TCM 
fractions and compounds, a dosage range was firstly estimated based on our previous experience [17-19]. Then the concentration was further adjusted according to their solubility in the embryo medium and their toxicity in zebrafish embryos. A concentration at which all fractions or compounds can be completely dissolved in the embryo medium, without causing obvious embryo toxicity was chosen for the screening. The toxicity assay of all compounds is shown in Additional file 2: Figure S1. The Phenylthiourea supplemented embryo medium was used to inhibit melanization as described before [20].

\section{High-throughput screening and data analysis}

2dpf $\operatorname{Tg}(L y z: D s R E D 2)$ transgenic embryos were dechorionated and dispensed in 96-well plate with 1 embryo/ well. Respective drug protection was supplemented in $300 \mu \mathrm{l}$ medium of each well at $2 \mathrm{dpf}$ and TNBS treatment was initiated at $3 \mathrm{dpf}$. At $5 \mathrm{dpf}$, embryos were incubated with $10 \mu \mathrm{M}$ DCFH-DA for 30-min staining in the dark to label ROS. Then the fish embryos were anesthetized in $0.016 \%$ Tricaine with side facing up, and automatically imaged by ImageXpress Micro Confocal (Molecular Devices, US.). The number of neutrophils and ROS level were also analyzed by the ImageXpress analyzing tools with the intestinal region manually outlined. At least 5 embryos were examined for each treatment condition. Two sets of cutoff value were determined to evaluate the drug effects. For the quantification of neutrophils accumulation, "Recovery rate of neutrophils accumulation" was calculated by $\left(\mathrm{N}_{\text {model }}-\mathrm{N}_{\text {drug }}\right) /\left(\mathrm{N}_{\text {model }}-\mathrm{N}_{\text {control }}\right)$. N represents for the number of neutrophils in the intestinal region, and a recovery rate $>0.6$ was deemed to be effective. For ROS levels, average fluorescence intensity less than $70 \%$ of the value of the model group after drug protection was considered as effective.

\section{Histological analysis}

5 dpf Zebrafish embryos were fixed in 4\% PFA at room temperature overnight, and were progressively dehydrated in ethanol. Embryos were embedded in paraffin blocks and were cut into $3 \mu \mathrm{m}$ sections and stained with hematoxylin and eosin. At least three embryos were examined for each treatment condition.

\section{QPCR}

Total RNA of zebrafish embryos was extracted by a RNAQuick Purification Kit (RN001, ES Science), and then converted to single- strand cDNA with HiFiScript cDNA Synthesis Kit (CW2569M, CWBIO). Real-time PCR was performed using the two-step quantitative RT-PCR method with 2 X SYBR Green qPCR Mater Mix (B21202, Bimake). The sequences of all primers used in the study are listed in Additional file 1: Table S2.

\section{Statistical analysis}

All data are presented as the mean \pm the standard error of the mean (SEM). Differences between two groups were analyzed using the two-tailed Student's t-test. Multiple group comparison was conducted by one-way ANOVA. An $p$ value $<0.05$ was considered statistically significant.

\section{Results \\ Knowledge mining of Zhongjing formulae}

Since Zhang Zhongjing created Treatise on Febrile Diseases at nearly 2000 years ago, it has been sorted out and edited numerous times by descendants. The formulae had also been clinically tested, and modified by following TCM doctors in many TCM handbooks, which were all referred as the classic Zhonging formulae. To avoid omissions, through careful literature review, we collected 40 ancient TCM books which are derived from or closely related to Zhongjing formulae, aside from the original book of Treatise on Febrile Diseases (Additional file 1: Table S1). Through word frequency analysis and vocabulary classification via Python, 248 TCM formulae were identified. A literature search was further conducted on China National Knowledge Infrastructure and NCBI Pubmed, using "Shanghan", "Zhongjing", or the names of the 248 Zhongjing formulae as keywords, in the time window between 1999/01/01 and 2018/12/31. In total, 79016 articles from CNKI and 85 articles from PubMed were additionally included for knowledge mining.

Knowledge mining was performed using the Word2vec model (Gensim, Python 3.6). Firstly, the word frequency of the text corpus was analyzed and the 100 most common words were retrieved (Fig. 1A). Noticeably, "enteritis", "colitis", "gastritis" were among the top hits of diseases or syndromes related to Zhongjing formulae, according to the frequency analysis results. Next, using the skipgram model of Word2vec, enteritis related disease names or syndromes were used as the input value, and all the meaningful words and phrases in the context were automatically located and retrieved as the output value. The degree of correlation was calculated based on the semantic distances between the input and output words, with cosine value labeled as the marker. The higher the cosine value is, the closer the relationship was suggested. Interestingly, words in different categories tend to be clustered in different subgroups when visualizing by $t$-distributed stochastic neighbor embedding, which suggested the reliability of the text training (Fig. 1B). As a result, we identified ten Zhongjing formulae most closely related to gastrointestinal inflammatory diseases, as recommended by knowledge mining (Fig. $1 \mathrm{C}$ and Additional file 1: Table S3). The results of knowledge mining were further verified in the other direction. The names of Zhongjing formulae identified in the previous step were used as the 


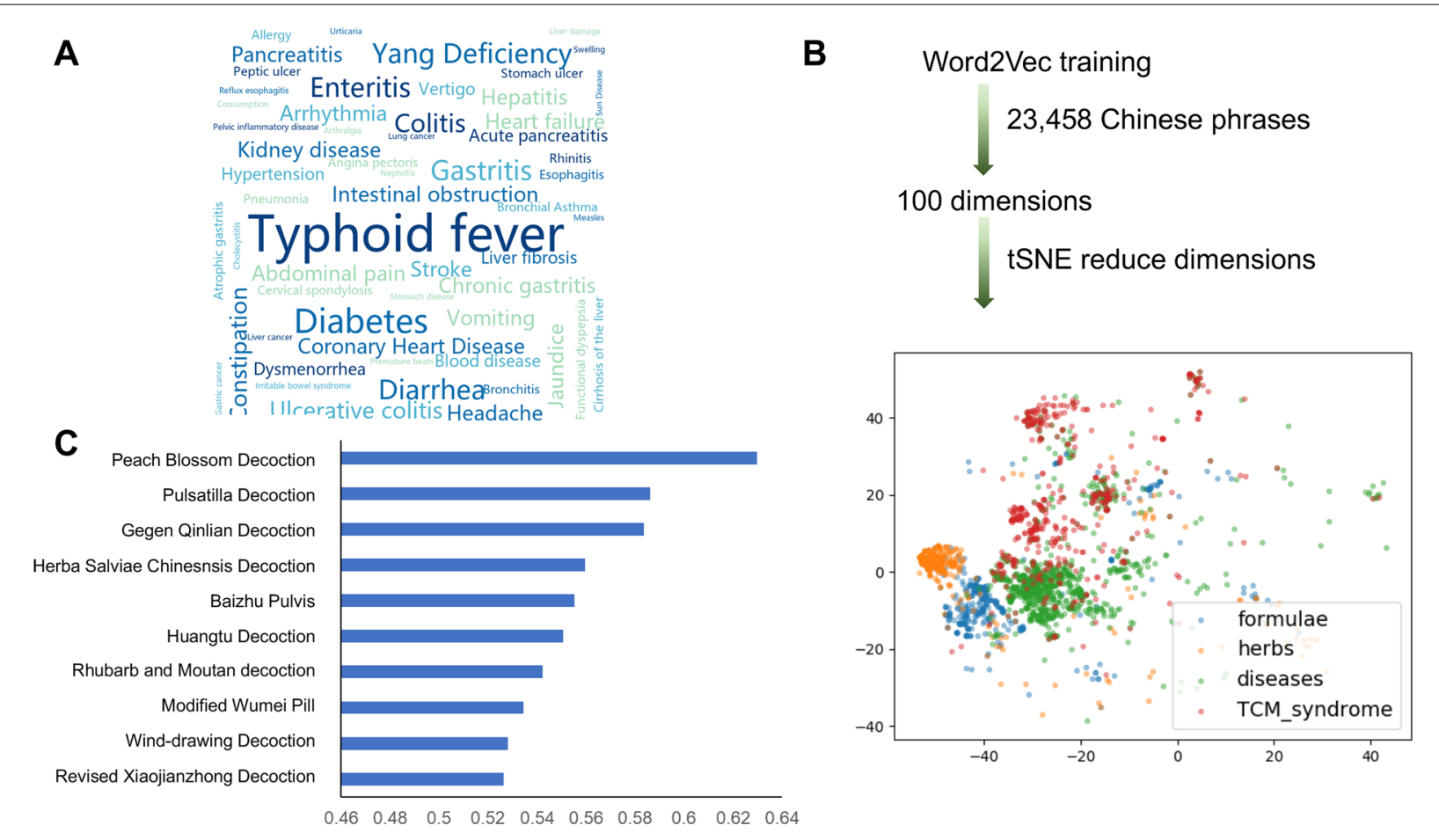

Fig. 1 Knowledge mining of Zhongjing formulae. A Word cloud of top 100 common diseases-related phrases in included literatures; B Flow chart of Word2Vec based knowledge mining analysis; C Ten Zhongjing formulae most closely related to gastrointestinal inflammatory diseases

input value, and the related symptoms or diseases in the context were predicted by the program. Consistently, a majority of symptoms related with these formulae are enteritis related, such as dysentery and diarrhea (Additional file 1: Table S4).

\section{TNBS induced typical IBD phenotypes in the zebrafish model}

Various IBD-like pathological changes were observed in TNBS-treated zebrafish in previous studies, such as intestinal morphological changes and increased neutrophils infiltration $[9,10]$. Consistently, in our study, increased number of neutrophils was observed in the intestines of $\operatorname{Tg}(L y z: D s R E D 2)$ transgenic fish, with fluorescein-labeled neutrophils under the lysozyme promoter [13], after $48 \mathrm{~h}$ stimulation in $75 \mathrm{ug} / \mathrm{ml}$ TNBS solution from $72 \mathrm{hpf}$ (Fig. 2A, B). Besides, as accumulating evidences suggested that intestinal ROS can accelerate epithelial cell damage and initiate IBD [21], the level of oxidative stress was examined in the TNBS-treated zebrafish with the DCFH-DA probe, which is a fluorescence indicator of the formation of $\mathrm{H}_{2} \mathrm{O}_{2}$ or other ROS and RNS [22]. As previously reported, a considerable level of ROS signal was detected in the intestine of wildtype zebrafish embryos using DCFH-DA, possibly due to high ROS generation during normal intestinal development [23]. TNBS stimulation showed a tendency to further increase the level of gastrointestinal ROS, although with no statistical significance (Fig. 2C, D). Nevertheless, co-treatment of 5-aminosalicyclic acid (5-ASA), a standard therapeutic drug of IBDs, significantly decreased both neutrophils accumulation and the ROS signal in TNBS-treated embryos. Moreover, histological analysis detected reduced intestinal folds, enlarged gut lumen and disrupted epithelial layer in zebrafish larvae treated with TNBS, suggesting decreased peristalsis and impaired mucosal barrier (Fig. 2E). Taken together, these findings suggested that TNBS treatment is capable of inducing IBD phenotypes in zebrafish, and this model was used for subsequent screens in our study.

\section{High-throughput screening of the fractions of formulae hits}

Based on the results of knowledge mining, three formulae with the highest likelihood to be related with IBD, as predicted by the Cosine value, were selected for further analysis, which were Peach Blossom Decoction (PBD), Pulsatilla Decoction (PD), and Gegen Qinlian Decoction (GQD) (Additional file 1: Table S2). Supportively, the therapeutic effects of these formulae in IBD and other 


\section{A}
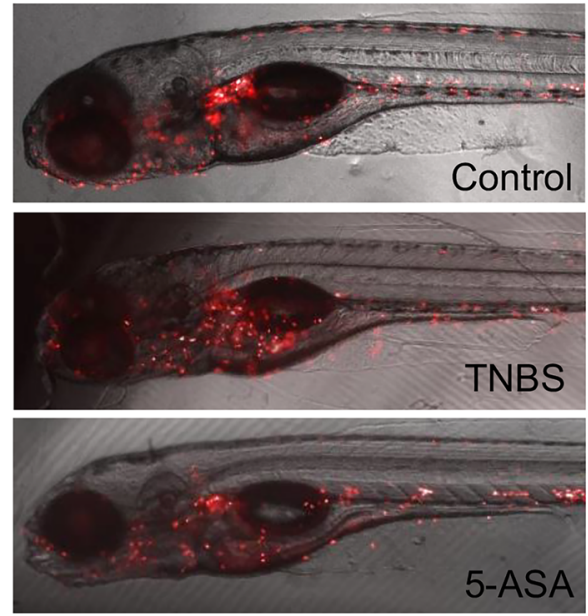

B

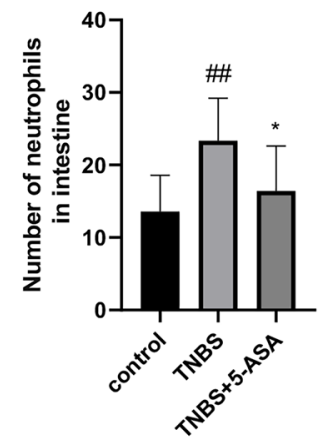

C

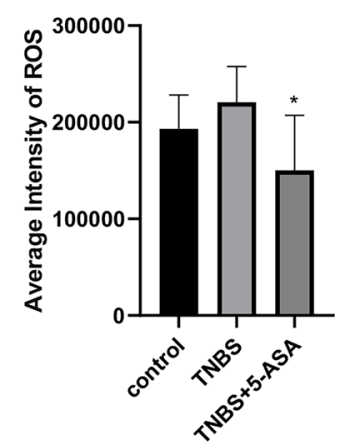

D
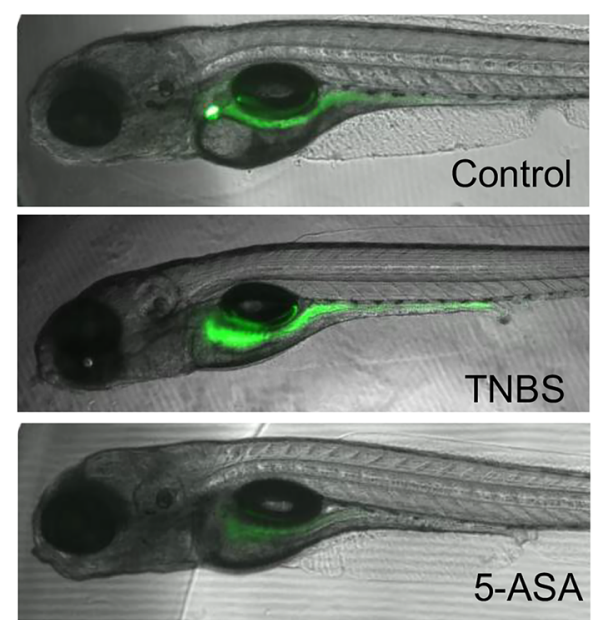

E

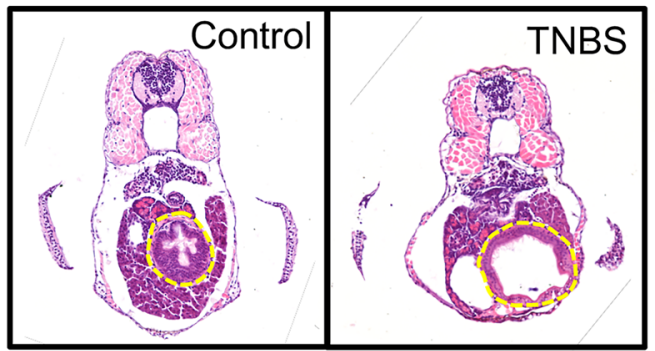

Fig. 2 TNBS induced IBD phenotypes in zebrafish. A, B The intestinal distribution of red fluorescent-labeled neutrophils in control, TNBS and 5-ASA treated embryos; at least 6 embryos were examined for each group. C, D ROS staining (green signals) in control, TNBS and 5-ASA treated embryos; at least 5 embryos were examined for each group. E HE staining of embryo cross sections. The intestine regions were circled by yellow dotted lines. Scale bar: $100 \mu \mathrm{m}$. \#compared with the control group; ${ }^{*}$ compared with the model group; \# or *, $p<0.05$, \#\# or **, $p<0.01$

enteritis were suggested by several other studies [24-26], but the active substances were still unclear. The fraction library of the three formulae was constructed as previously reported [17], which includes 74 fractions in total. Next, using the TNBS-induced zebrafish IBDs model, the therapeutic effects of these TCM fractions were screened. TCM fractions were supplemented to zebrafish embryos, at a dosage of $50 \mu \mathrm{g} / \mathrm{mL}$, from $24 \mathrm{~h}$ before the TNBS treatment till the imaging analysis. The number of neutrophils and the level of ROS signals in the intestinal tract were used as the output indexes for efficacy evaluation. Using the performance of 5-ASA as a reference, two sets of cutoff values were used to facilitate efficient screening (see "Materials and methods" for details). As a result, 36 fractions were found to be effective for at least one marker, and six of them showed regulation on both markers (Fig. 3A, B). Interestingly, although five PBD fractions strongly inhibited the intestinal neutrophils accumulation, their effects on ROS signal were not significant. On the contrary, among the six fractions influence both neutrophils accumulation and ROS level, five fractions were derived from GQD. These findings suggested that the therapeutic effects of different TCM formulae rely on different pharmacological routes. In the current study, the six fractions with dual function on regulating neutrophils and ROS were included for the subsequent composition analysis and validation assay.

\section{Identification of main chemical compounds in active fractions by mass spectrometry}

Next, the chemical components in the six fractions which showed regulatory effects on both neutrophils accumulation and endogenous ROS level were analyzed by LC-MS in both negative and positive modes (Fig. 4 and Additional file 3: Figure S2). After examining the raw data, main peaks were identified in the fractions as major compounds. The molecular formulae of these compounds were obtained and the candidate structures were inferred from literatures and public databases. Based on the MS results, 7 compounds from GQD and 6 compounds from 

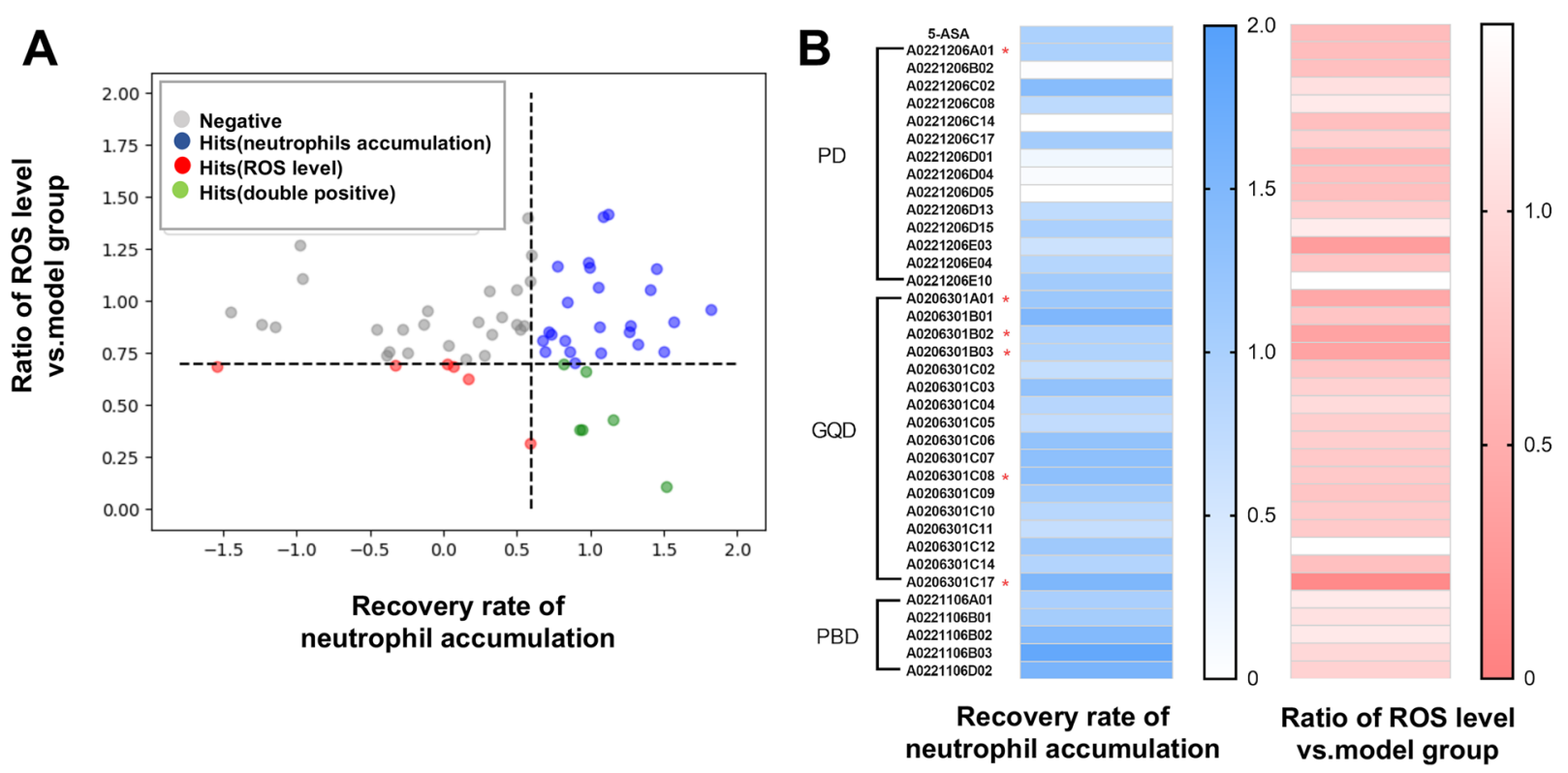

Fig. 3 Results of high-content screening of Zhongjing formulae fractions. A Scatter plot showing the effects of 74 formulae on intestinal neutrophils accumulation and ROS. B Heat map showing the rescue levels of 36 formulae with significant effects at at least one screening criterion. The formulae with dual effects in regulating both neutrophil number and ROS are marked with a red asterisk

PD were selected for further identification, by comparisons with reference standards in terms of retention time and mass spectra. Among these compounds, 3 compounds were suggested to originate from the plant Radix Puerariae, 3 from Scutellaria Baicalensis, 2 from Cortex Fraxini, 2 from Coptis Chinensis Franch, 2 from Phellodendron Chinense Schneid, and 1 from Glycyrrhiza Uralensis Fisch. The chemical class of the potential active compounds includes flavonoids, saponins, alkaloids, phenolic acid and coumarins. The potential functions for these compounds by previous studies are summarized in Table 1, almost all of which were involved in the regulation of inflammatory processes.

\section{Validation of active compounds with anti-IBD effects}

Based on the results of HRMS, the above 13 compounds were included to further evaluate their regulatory effects on the development of intestinal inflammation in the TNBS-induced zebrafish IBDs model. Similar with the efficacy screening conducted for TCM fractions, the solution of each compound was supplemented to the embryo medium from $24 \mathrm{~h}$ before TNBS treatment till the end of the analysis, at a concentration of $200 \mu \mathrm{M}$. As a result, a total of 6 compounds showed protective effects against intestinal inflammation when using neutrophil accumulation or ROS level as readouts. Specifically, daidzin, palmatine, oroxyloside, chlorogenic acid, and aesculin significantly suppressed the accumulation of neutrophils in the intestinal tracts of fish IBD model, with daidzin and palmatine showing the most prominent effects (Fig. 5A, B). Besides, baicalin and oroxyloside are sufficient to downregulate the intestinal level of ROS when used alone (Fig. 5C, D). Oroxyloside, a flavonoid compound from the plant Scutellaria Baicalensis, was the only compound which displays moderate, but significant effects in the regulation of both neutrophil accumulation and oxidative stress. After literature review, we found more evidences in the inflammation regulation of the above compound hits from cell line or murine models [27-32], which suggests the reliability of our screening system. In addition, the regulation effect of daidzin in intestinal inflammation was novelly identified in our screen, which could be an interesting therapeutic candidate of IBD.

(See figure on next page.)

Fig. 4 Component analysis of effective fractions identified in the screen. A, B Base peak chromatogram of fraction A0206301B03 of Gegen Qinlian Decoction (A) and fraction A0221206A01 of Pulsatilla Decoction (B) obtained by UPLC-Q-TOF in positive ion mode. The name of chemical compounds identified with reference standards are labeled. C Comparison of main chemical compounds with reference standards 


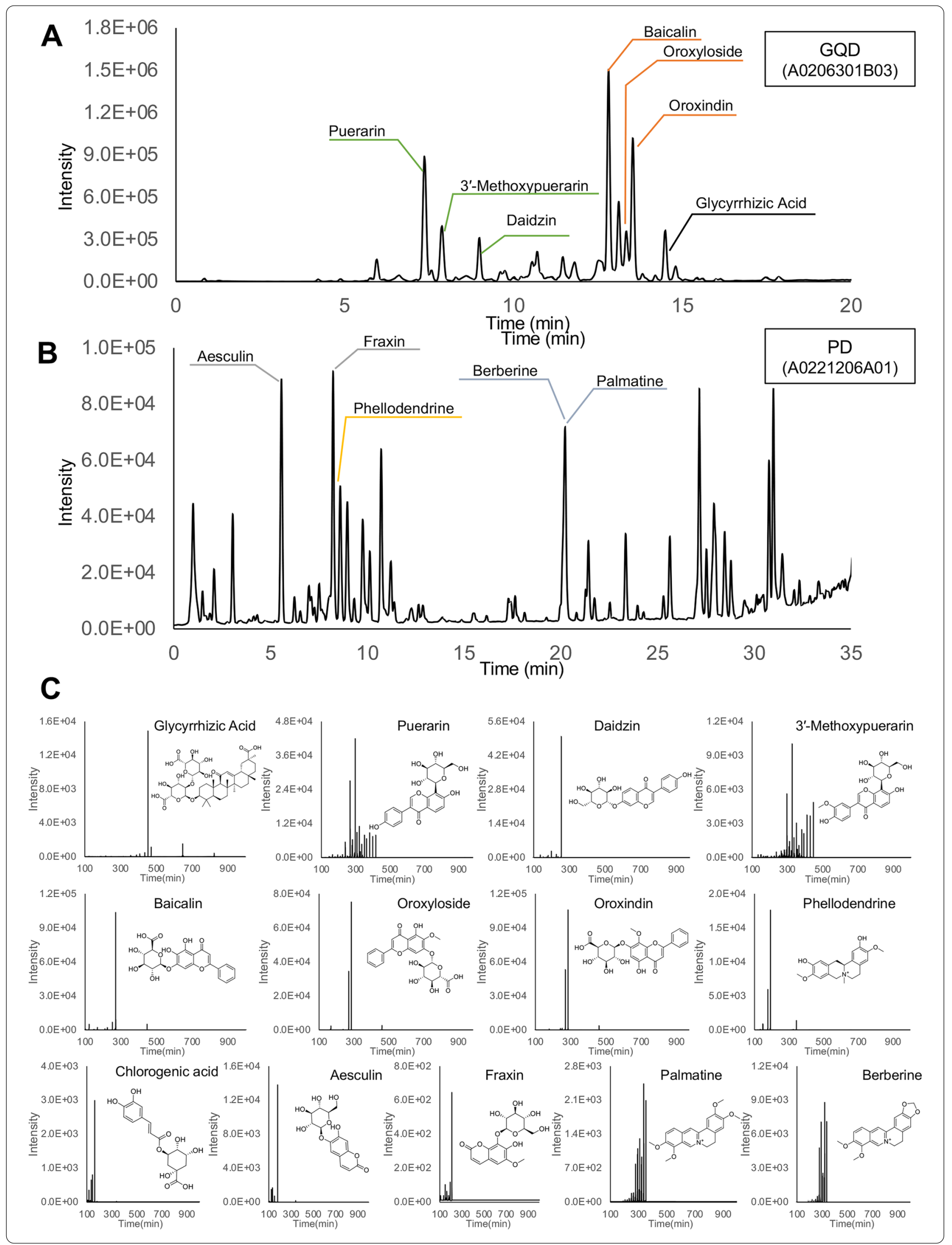


Table 1 Summary of previously reported functions of compounds hits

\begin{tabular}{|c|c|c|c|}
\hline Compound & Formula & Herbal origins & Suggested function \\
\hline Baicalin & GQD & Scutellaria Baicalensis & $\begin{array}{l}\text { Cardioprotection [45], enhancing glucose uptake and insulin sensitivity [46], anti- } \\
\text { inflammation, anti-bacterial infection [47], anti-tumor [48], anti-oxidative stress, } \\
\text { anti-allergy [49] }\end{array}$ \\
\hline Daidzin & GQD & Radix Puerariae & Anti-tumor [50], anti-inflammation [51], anti-oxidative stress [52] \\
\hline 3'-Methoxypuerarin & GQD & Radix Puerariae & Hepatoprotective [53] \\
\hline Oroxindin & GQD & Scutellaria Baicalensis & $\begin{array}{l}\text { Anti-inflammation, anti-tumor, anti-oxidative stress [54], anti-bacterial infection, } \\
\text { anti-proliferation [55] }\end{array}$ \\
\hline Glycyrrhizic acid & GQD & Glycyrrhiza Uralensis Fisch & $\begin{array}{l}\text { Anti-viral infection [56], anti-inflammation [57], hepatoprotective [58], anti-cancer, } \\
\text { anti-ulcer, anti-anaphylaxis [59] }\end{array}$ \\
\hline Puerarin & GQD & Radix Puerariae & $\begin{array}{l}\text { Vasodilation, cardio-protective [60], neuroprotection, anti-oxidation, anti-inflamma- } \\
\text { tion [61], anti-cancer [62], alleviating pain, promoting bone formation, inhibiting } \\
\text { alcohol uptake [63], attenuating insulin resistance [60] }\end{array}$ \\
\hline Oroxyloside & GQD & Scutellaria Baicalensis & $\begin{array}{l}\text { Inhibiting angiogenesis [64], anti-cancer [65], hepatoprotective [66], anti-inflamma- } \\
\text { tion [29] }\end{array}$ \\
\hline Esculin & PD & Cortex Fraxini & $\begin{array}{l}\text { Hepatoprotective [67], anti-bacterial infection, anti-inflammation [68], anti-allergy, } \\
\text { skin protection [69], anti-thrombosis, anti-oxidative stress [70] }\end{array}$ \\
\hline Fraxin & PD & Cortex Fraxini & Hepatoprotective, anti-oxidative stress [71], anti-inflammation [72] \\
\hline Phellodendrine & PD & Phellodendron Chinense Schneid & Anti-oxidative stress, anti-inflammation, reducing blood pressure [73] \\
\hline Palmatine & PD & Coptis Chinensis Franch & $\begin{array}{l}\text { Anti-cancer, anti-oxidation, anti-inflammation, neuroprotection, anti-bacterial and } \\
\text { virus infection, regulating blood lipids [74] }\end{array}$ \\
\hline Berberine & PD & Coptis Chinensis Franch & $\begin{array}{l}\text { Anti-microbial infection, anti-diabetis, anti-cancer, lipid-lowering, anti-diarrhea, } \\
\text { antitrachoma, anti-oxidation, anti-inflammation, antiviral infection [75-77] }\end{array}$ \\
\hline Chlorogenic acid & PD & Phellodendron Chinense Schneid & $\begin{array}{l}\text { Anti-oxidative stress, anti-microbial infection, hepatoprotective, cardioprotective, } \\
\text { anti-inflammation, antipyretxia, neuroprotection, anti-obesity, anti-hypertension, } \\
\text { anti-oxidative stress, neuronal stimulation [78-81] }\end{array}$ \\
\hline
\end{tabular}

\section{The impacts of active compounds on the expression} of inflammation and intestinal barrier markers

Finally, we examined the potential influences of the Zhongjing formulae compounds on the endogenous expression of factors related to IBD development. After TNBS stimulation, the transcriptional level of multiple inflammatory related factors, including il $1 \beta$, chemokine (C-X-C motif) ligand 8a ( $x$ cl $8 a)$, matrix metallopeptidase $9(m m p 9)$, and $t n f \alpha$ were all significantly increased (Fig. 6A, B). Noticeably, all the compounds displayed potent inhibitory effects on the expression of $i l 1 \beta$, supporting their anti-inflammatory effects. Moreover, aesculin also significantly inhibited the mRNA levels of cxcl8a, mmp 9 and $t n f-\alpha$. In order to analyze the effects of these compounds on intestinal barrier, the expression of adherens junction protein E-Cadherin and the epithelial transcription factor hepatocyte nuclear factor $4 \alpha(h n f 4 \alpha)$ were also examined. Importantly, the transcriptional level of e-cadherin was upregulated by 5-ASA, daidzin and aesculin, suggesting that these agents may function through protecting the integrity of intestinal epithelial layer. We further inspected intestinal phenotypes directly by histological analysis. Compared with pathological alterations in the TNBS treated embryos, the intestinal morphology was largely maintained in all compoundtreated groups (Fig. 6C).

\section{Discussion}

Since the pathogenesis of IBD is still elusive, current treatment approaches of the disease are mostly symptomatically targeted, which aim to inhibit the progression of chronic intestinal inflammation. General inflammatory suppressant, such as corticosteroids and aminosalicylates, are routine IBD drugs. In recent years, the introduction of anti-TNF agents initiated a new era in IBD treatment. Infliximab and adalimumab can effectively induce clinical remission and mucosal healing in IBD patients [33]. However, one third of IBD patients failed to respond to anti-TNF treatment and $40 \%$ of initial responders developed intolerance over a year $[34,35]$. Other biological immunomodulators, such as ustekinumab, an mAb against IL12/IL23, showed efficiency in moderately to severely active Crohn's diseases, and brought clinical benefits in many patients who had failed anti-TNF treatment [36, 37]. Nevertheless, still more than $1 / 3$ of the IBD patients showed no response to ustekinumab during the first year [38], and limited endoscopic remission was observed in ustekinumab treated patients after 24 weeks [36]. Therefore, ongoing drug development and mechanism research are needed for the management of IBD.

According to the TCM theory, specific combinations of herbs or other naturally-derived medicinal materials 


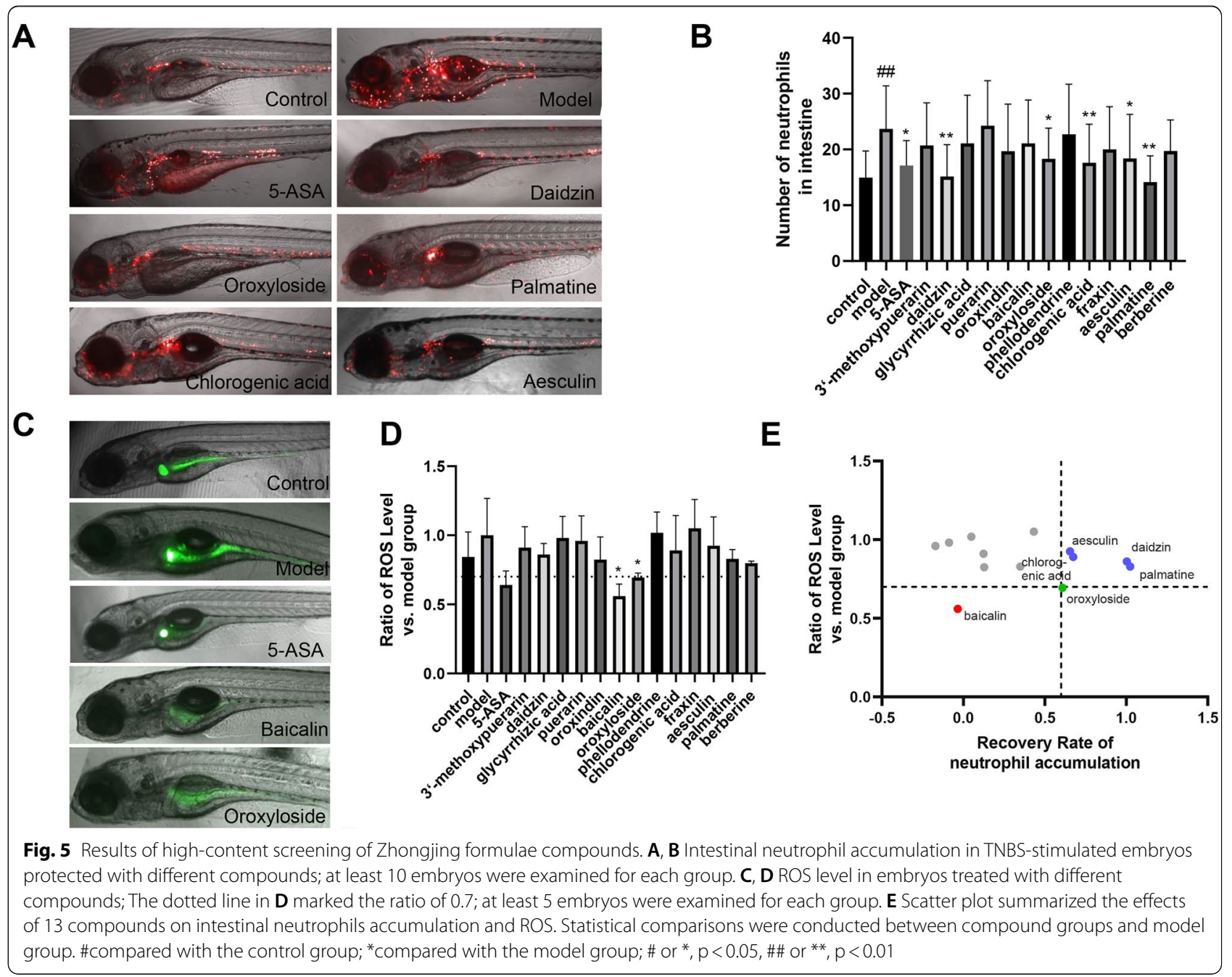

can generate cooperative or synergistic effects in treating particular diseases. Indeed, the therapeutic efficacies of many TCM formulae have been continuously testified by millennial clinical practices. However, after being modified by TCM doctors of many generations, the total amount of TCM formulae becomes very large, yet the descriptions of many formulae were only recorded in ancient languages, which made it difficult to review for nowadays researchers. On the other hand, dozens or even hundreds of compounds can be detected in even one herb, not to mention combinations of multiple herbs in a typical TCM formula. Thus, it is particularly challenging to identify the effective components. As a result, our understanding of TCM formulae was driven into a stagnant status for decades. Nevertheless, with the rapid development in modern techniques, re-examining the hidden secrets of TCM formulae may become possible.

Here, we innovatively combined the approaches of knowledge mining, high-content analysis and HRMS, to systematically identify effective TCM formulae and compounds in IBD treatment. Due to the large amount of healthcare data, computerized knowledge mining has been an intriguing area of medical research [39]. In order to unbiasedly distinguish TCM formulae related to IBD characteristic symptoms, both ancient and modern literatures of Zhongjing formulae, which is a large collection of TCM prescriptions with most abundant empirical evidences, were collected in our study, and the Word2vec algorithm was used to perform analogy retrieval and semantic relation retrieval [15]. As a result, PBD, PD and GQD were identified as the three formulae with the closest relationship with IBD. A previous randomized control trial demonstrated superior therapeutic effect of PBD in ulcerative colitis patients, compared with 5-ASA and glucocorticoid, with reduced levels of TNF- $\alpha$ and IL-8 [40]. PD administration attenuated the severity of colitis signs in mice induced by oxazolone or dextran sulfate 


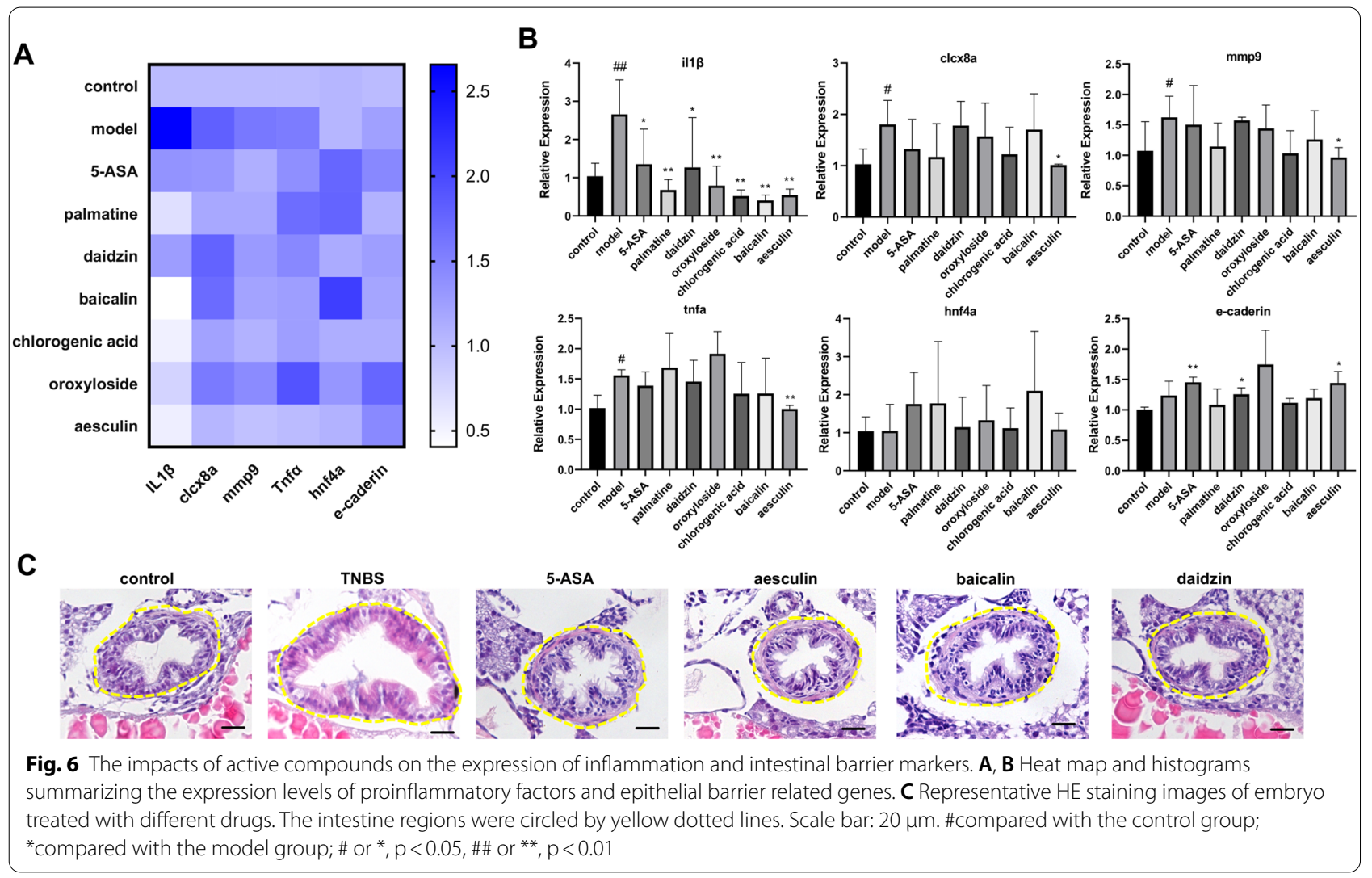

sodium(DSS), which significantly reduced the secretion of pro-inflammatory cytokines and improved the colonic pathological manifestations [26, 41]. GQD oral administration was found to alleviate the severity of colitis in DSS-induced mice model, with reduced tolllike receptor 4 expression and NF- $\mathrm{KB}$ activation, as well as decreased level of pro-inflammatory cytokines [24]. Another study reported opposite changes of Notch signaling in acute and chronic ulcerative colitis mice models after DSS stimulation, whereas GQD was able to exhibit bidirectional regulation on Notch signaling [25]. Consistently, multiple fractions of the three TCM decoctions were found to exhibit significant regulatory effects in the zebrafish IBD model. Noticeably, despite being predicted as the top one TCM formula linked to IBD symptoms by knowledge mining, the fractions of PBD only showed significant effects in suppressing neutrophils recruitment, but not the endogenous ROS level, suggesting that its pharmacological mechanism may be different from PD and GQD. Other inflammation related markers and signaling pathways should be further examined to elaborate the molecular mechanism of these TCM decoctions in IBD treatment.

In the zebrafish IBD model, we further evaluated the regulatory effects of representative compounds. The zebrafish digestive system undergoes rapid development during early stages. In zebrafish embryos, a continuous gut tube formed at 2 to $3 \mathrm{dpf}$, and the mouth opened at about $4 \mathrm{dpf}$. By $5 \mathrm{dpf}$, the digestive tract can support the feeding and digestion processes, with the formation of many digestive organs functionally remseble their mammalian counterparts [42]. Based on previous experiences of others and our group $[18,19,43]$, through soaking the zebrafish embryos in compounds supplemented growth medium, most chemical compounds can efficiently enter the blood circulation and digestive system of zebrafish embryos by direct skin penetration or absorption via mouthes or gills. Nevertheless, the rates of absorption and the pharmacodynamic characteristics of different drugs in zebrafish embryo are still unlcear, which may influence the quantitative evaluation of drug effects. Among the identified chemical compounds in our study, aesculin and daidzin are of particular interests to us. Aesculin is a coumarin compounds derived from Cortex fraxini, a main herb in PD. The protective effects of aesculin in DSS-induced colitis in mice have been reported previously, which may be mediated through the PPAR- $\gamma$ and NF-kB pathway [30]. Consistently, aesculin rescued intestinal inflammation phenotype and restrained the upregulation of multiple inflammatory factors $(i l 1 \beta, \operatorname{tnf} \alpha$, 
clcx8a, and mmp9) in our screening, suggesting its potent effect in inflammation regulation. Moreover, increased expression of e-cadherin was also observed in aesculin treated embryos. The adherens junctions of intestinal epithelial cells are formed mainly via E-cadherin, which is essential for the maintenance of intestinal barrier. As reported previously, knocking down e-cadherin led to severely aggravated symptoms of experimental colitis were in mice [44]. In addition, we also reported the protective effects of daidzin, an isoflavonoids compound derived from Radix Puerariae, in intestinal inflammation for the first time. Dramatically decreased neutrophil accumulation in the fish gut was observed in daidzin treated group, along with inhibited il1 $\beta$ expression and upregulated $e$-cadherin transcription. The positive regulation of aesculin and daidzin on e-cadherin's expression, on top of their anti-intestinal inflammation effects, making them inviting IBD drug candidates.

Limitations remain existed in our study. For the current attempt of high-content screening, we only used two sets of screening outputs, intestinal neutrophils accumulation and ROS level, which may result in the omission of formulae or compounds that do not function through these two pathological processes. Some modifications can be made in future studies. For example, by using intestinal epithelial cells labeled transgenic fish lines, the intestinal morphological changes can be directly observed and applied as a golden standard for the screening. Besides, other fluorescent probes for key proinflammatory factors can also be explored in the screen, to identify compound with specific regulatory effects on a particular target protein.

\section{Conclusion}

Aiming at the drug development needs in IBD treatment, we performed a systematic and unbiased screening in Zhongjing formulae via combining the techniques of knowledge mining, live embryo-based high-content analysis, and high-resolution mass spectrometry. The regulatory effects of TCM formulae fractions and representative compounds were demonstrated in our study, with aesculin and daidzin being particularly interesting IBD drug candidates. Future studies are warranted to further investigate the downstream targets and pharmacological mechanism of these TCM formulae and compounds in IBD treatment. The research strategy in our study can also be applied in the discovery of effective TCM formulae and components in other diseases.

\section{Abbreviations}

Cxcl8a: Chemokine (C-X-C motif) ligand 8a; GQD: Gegen Qinlian Decoction; hnf4a: Hepatocyte nuclear factor 4a; HRMS: High-resolution mass spectrometry; IBD: Inflammatory bowel diseases; IL: Interleukin; mAbs: Monoclonal antibodies; mmp9: Matrix metallopeptidase 9; PBD: Peach Blossom Decoction; PD: Pulsatilla Decoction; ROS: Reactive oxygen species; TCM: Traditional Chinese medicine; TNBS: Trinitrobenzene sulfonic acid; TNF-a:Tumor necrosis factor-a; 5-ASA: 5-Aminosalicyclic acid.

\section{Supplementary Information}

The online version contains supplementary material available at https://doi. org/10.1186/s13020-021-00452-z.

Additional file 1: Table S1. List of ancient Zhongjing Formula related ancient TCM books included for the knowledge mining. Table S2. List of QPCR primers used in this study. Table S3. List of top ranking Zhongjing formulae related to inflammatory bowel diseases based on the results of knowledge mining. Table S4. List of top ranking syndromes related to the IBD-related Zhongjing formulae based on the results of knowledge mining.

Additional file 2: Figure S1. Toxicity assay of all compounds in the screening.

Additional file 3: Figure S2. Base peak chromatogram of all fraction hits obtained by UPLC-Q-TOF in negative (NEG) or positive(POS) ion modes.

\section{Acknowledgements}

The authors are grateful for the support from ZJU PII-Molecular Devices Joint Laboratory and ZJU PII-Tecan Joint Laboratory.

\section{Authors' contributions}

Conception and design: LZ and YW. Acquisition of data: YY, JC, XZ, YW and SW. Analysis and interpretation of data: $Y Y, J C, X Z$. Writing of the manuscript: $L Z, Y Y$, and $Y$ W. All authors read and approved the final manuscript.

\section{Funding}

This work was supported by National Natural Science Foundation of China (Grant no. 31971088, 81822047), National Key R\&D Program of China (no. 2018YFC1704502), and the Zhejiang Key Agricultural Enterprise Institute (2017Y20001)

\section{Availability of data and materials}

The datasets used during the current study are available from the corresponding author on reasonable request.

\section{Declarations}

\section{Ethics approval and consent to participate}

The study was review and approved by the Animal Ethics Committee of the Laboratory Animal Center, Zhejiang University.

\section{Consent for publication}

Not applicable.

\section{Competing interests}

We wish to confirm that there are no known conflicts of interest associated with this publication and there has been no significant financial support for this work that could have influenced its outcome.

\section{Author details}

${ }^{1}$ Pharmaceutical Informatics Institute, College of Pharmaceutical Sciences, Zhejiang University, Hangzhou 310058, China. ${ }^{2}$ Innovation Institute for Artificial Intelligence in Medicine of Zhejiang University, Hangzhou 310058, China. ${ }^{3}$ State Key Laboratory of Component-Based Chinese Medicine, Tianjin University of Traditional Chinese Medicine, Tianjin, China.

Received: 2 March 2021 Accepted: 24 May 2021

Published online: 31 May 2021 


\section{References}

1. Neurath MF. Cytokines in inflammatory bowel disease. Nat Rev Immunol. 2014;14:329-42.

2. Ng SC, Shi HY, Hamidi N, Underwood FE, Tang W, Benchimol El, et al. Worldwide incidence and prevalence of inflammatory bowel disease in the 21 st century: a systematic review of population-based studies. Lancet (London, England). 2018;390:2769-78.

3. Plichta DR, Graham DB, Subramanian S, Xavier RJ. Therapeutic opportunities in inflammatory bowel disease: mechanistic dissection of host-microbiome relationships. Cell. 2019;178:1041-56.

4. Tu Y. The discovery of artemisinin (qinghaosu) and gifts from Chinese medicine. Nat Med. 2011;17:1217-20.

5. Zhang C, Jiang M, Lu A. Considerations of traditional Chinese medicine as adjunct therapy in the management of ulcerative colitis. Clin Rev Allergy Immunol. 2013:44:274-83.

6. Guo BJ, Bian ZX, Qiu HC, Wang YT, Wang Y. Biological and clinical implications of herbal medicine and natural products for the treatment of inflammatory bowel disease. Ann NY Acad Sci. 2017:1401:37-48.

7. Sugimoto K, Hanai H, Tozawa K, Aoshi T, Uchijima M, Nagata T, et al. Curcumin prevents and ameliorates trinitrobenzene sulfonic acid-induced colitis in mice. Gastroenterology. 2002;123:1912-22.

8. Wirtz S, Neurath MF. Mouse models of inflammatory bowel disease. Adv Drug Deliv Rev. 2007;59:1073-83.

9. Fleming A, Jankowski J, Goldsmith P. In vivo analysis of gut function and disease changes in a zebrafish larvae model of inflammatory bowel disease: a feasibility study. Inflamm Bowel Dis. 2010;16:1162-72.

10. Oehlers SH, Flores MV, Hall CJ, Okuda KS, Sison JO, Crosier KE, et al. Chemically induced intestinal damage models in zebrafish larvae. Zebrafish. 2013;10:184-93.

11. Zhou GX, Liu ZJ. Potential roles of neutrophils in regulating intestinal mucosal inflammation of inflammatory bowel disease. J Dig Dis. 2017;18:495-503.

12. Bhattacharyya A, Chattopadhyay R, Mitra S, Crowe SE. Oxidative stress: an essential factor in the pathogenesis of gastrointestinal mucosal diseases. Physiol Rev. 2014:94:329-54.

13. Hall C, Flores MV, Storm T, Crosier K, Crosier P. The zebrafish lysozyme C promoter drives myeloid-specific expression in transgenic fish. BMC Dev Biol. 2007:7:42.

14. Westerfield M. The Zebrafish Book. A guide for the Laboratory Use of Zebrafish (Danio Rerio). fourth. Eugene, editor. Univ. of Oregon Press; 2000

15. Rong X. word2vec parameter learning explained. arXiv Prepr arXiv. 2014; http://arxiv.org/abs/1411:2738.

16. Lilleberg J, Zhu Y, Zhang Y. Support vector machines and Word2vec for text classification with semantic features. In: Proceedings of the 2015 IEEE 14th international conference on cognitive informatics and cognitive computing $\mid \mathrm{CCI}^{*} \mathrm{CC}$ 2015. New York: IEEE; 2015. p. 136-40.

17. Wang S, Wang H, Liu Y, Wang Y, Fan X, Cheng Y. Rapid discovery and identification of anti-inflammatory constituents from traditional Chinese medicine formula by activity index, LC-MS, and NMR. Sci Rep. 2016:6:31000

18. Li J, Liu H, Yang Z, Yu Q, Zhao L, Wang Y. Synergistic effects of cryptotanshinone and senkyunolide I in guanxinning tablet against endogenous thrombus formation in zebrafish. Front Pharmacol. 2020;11:622787.

19. Sheng J, Meng Q, Yang Z, Guan J, Zhao Y, Zhang J, et al. Identification of cryptotanshinone from Tongmai to inhibit thrombosis in zebrafish via regulating oxidative stress and coagulation cascade. Phytomedicine. 2020;76:153263.

20. Karlsson J, von Hofsten J, Olsson PE. Generating transparent zebrafish: a refined method to improve detection of gene expression during embryonic development. Mar Biotechnol (NY). 2001;3:522-7.

21. Tian T, Wang Z, Zhang J. Pathomechanisms of oxidative stress in inflammatory bowel disease and potential antioxidant therapies. Oxid Med Cell Longev. 2017;2017:4535194.

22. Gomes A, Fernandes E, Lima JLFC. Fluorescence probes used for detection of reactive oxygen species. J Biochem Biophys Methods. 2005;65:45-80.

23. Shi Y, Zhang Y, Zhao F, Ruan H, Huang H, Luo L, et al. Acetylcholine serves as a derepressor in Loperamide-induced Opioid-Induced Bowel Dysfunction (OIBD) in zebrafish. Sci Rep. 2014;4:5602.
24. Li R, Chen Y, Shi M, Xu X, Zhao Y, Wu X, et al. Gegen Qinlian decoction alleviates experimental colitis via suppressing TLR4/NF-KB signaling and enhancing antioxidant effect. Phytomedicine. 2016;23:1012-20.

25. Zhao Y, Luan H, Gao H, Wu X, Zhang Y, Li R. Gegen Qinlian decoction maintains colonic mucosal homeostasis in acute/chronic ulcerative colitis via bidirectionally modulating dysregulated Notch signaling. Phytomedicine. 2020;68:153182

26. Zhang W, Chen Y, Jiang H, Yang J, Wang Q, Du Y, et al. Integrated strategy for accurately screening biomarkers based on metabolomics coupled with network pharmacology. Talanta. 2020;211:120710.

27. Zhang X-J, Yuan Z-W, Qu C, Yu X-T, Huang T, Chen PV, et al. Palmatine ameliorated murine colitis by suppressing tryptophan metabolism and regulating gut microbiota. Pharmacol Res. 2018;137:34-46.

28. Zhang Z, Wu X, Cao S, Cromie M, Shen Y, Feng Y, et al. Chlorogenic acid ameliorates experimental colitis by promoting growth of akkermansia in mice. Nutrients. 2017:9:677.

29. Wang $X$, Sun $Y$, Zhao $Y$, Ding $Y$, Zhang $X$, Kong $L$, et al. Oroxyloside prevents dextran sulfate sodium-induced experimental colitis in mice by inhibiting NF-kB pathway through PPARy activation. Biochem Pharmacol. 2016:106:70-81.

30. Tian X, Peng Z, Luo S, Zhang S, Li B, Zhou C, et al. Aesculin protects against DSS-Induced colitis though activating PPARY and inhibiting NF-кВ pathway. Eur J Pharmacol. 2019;857:1753.

31. Shen J, Cheng J, Zhu S, Zhao J, Ye Q, Xu Y, et al. Regulating effect of baicalin on IKK/IKB/NF-kB signaling pathway and apoptosis-related proteins in rats with ulcerative colitis. Int Immunopharmacol. 2019;73:193-200.

32. Zhang C-L, Zhang S, He W-X, Lu J-L, Xu Y-J, Yang J-Y, et al. Baicalin may alleviate inflammatory infiltration in dextran sodium sulfate-induced chronic ulcerative colitis via inhibiting IL-33 expression. Life Sci. 2017;186:125-32

33. Gecse KB, Lakatos PL. IBD in 2016: Biologicals and biosimilars in IBDthe road to personalized treatment. Nat Rev Gastroenterol Hepatol. 2017:14:74-6.

34. Ben-Horin S, Chowers Y. Review article: loss of response to anti-TNF treatments in Crohn's disease. Aliment Pharmacol Ther. 2011;33:987-95.

35. Roda G, Jharap B, Neeraj N, Colombel J-F. Loss of response to anti-TNFs: definition, epidemiology, and management. Clin Transl Gastroenterol. 2016;7:e135.

36. Verstockt B, Dreesen E, Noman M, Outtier A, Van den Berghe N, Aerden l, et al. Ustekinumab exposure-outcome analysis in Crohn's disease only in part explains limited endoscopic remission rates. J Crohns Colitis. 2019:13:864-72.

37. Amiot A, Grimaud J-C, Peyrin-Biroulet L, Filippi J, Pariente B, Roblin X, et al. Effectiveness and Safety of Vedolizumab Induction Therapy for Patients With Inflammatory Bowel Disease. Clin Gastroenterol Hepatol Off Clin Pract J Am Gastroenterol Assoc. 2016:14:1593-601.

38. Wils P, Bouhnik Y, Michetti P, Flourie B, Brixi H, Bourrier A, et al. Longterm efficacy and safety of ustekinumab in 122 refractory Crohn's disease patients: a multicentre experience. Aliment Pharmacol Ther. 2018;47:588-95.

39. Chen ES, Sarkar IN. Mining the electronic health record for disease knowledge. Methods Mol Biol. 2014;1159:269-86.

40. Lei N, Kong $P$, Chen S, Tang X. Regulatory effect of huaihuasan combined with taohuatang on immune inflammation during active period of ulcerative colitis with cold-heat syndrome. Chin J Exp Trad Med Formulae. 2020;26:86-91.

41. Wang X, Fan F, Cao Q. Modified Pulsatilla decoction attenuates oxazolone-induced colitis in mice through suppression of inflammation and epithelial barrier disruption. Mol Med Rep. 2016;14:1173-9.

42. Yang $Y$, Tomkovich S, Jobin C. Could a swimming creature inform us on intestinal diseases? Lessons from zebrafish Inflamm Bowel Dis 2014:20:956-66.

43. Ridges S, Heaton WL, Joshi D, Choi H, Eiring A, Batchelor L, et al. Zebrafish screen identifies novel compound with selective toxicity against leukemia. Blood. 2012;119:5621-31.

44. Grill Jl, Neumann J, Hiltwein F, Kolligs FT, Schneider MR. Intestinal E-cadherin deficiency aggravates dextran sodium sulfate-induced colitis. Dig Dis Sci. 2015;60:895-902. 
45. Yu H, Chen B, Ren Q. Baicalin relieves hypoxia-aroused H9c2 cell apoptosis by activating Nrf2/HO-1-mediated HIF1a/BNIP3 pathway. Artif Cells Nanomed Biotechnol. 2019;47:3657-63.

46. Fang P, Sun Y, Gu X, Shi M, Bo P, Zhang Z, et al. Baicalin ameliorates hepatic insulin resistance and gluconeogenic activity through inhibition of p38 MAPK/PGC-1 a pathway. Phytomedicine. 2019;64:153074.

47. Ji W, Liang K, An R, Wang X. Baicalin protects against ethanol-induced chronic gastritis in rats by inhibiting Akt/NF-kB pathway. Life Sci. 2019:239:117064

48. Xu W-F, Liu F, Ma Y-C, Qian Z-R, Shi L, Mu H, et al. Baicalin regulates proliferation, apoptosis, migration, and invasion in mesothelioma. Med Sci Monit Int Med J Exp Clin Res. 2019:25:8172-80.

49. Ma J, Wang $R$, Yan $H, X u R, X u A$, Zhang J. Protective effects of baicalin on lipopolysaccharide-induced injury in Caenorhabditis elegans. Pharmacology. 2020;105:109-17

50. Yang MH, Jung SH, Chinnathambi A, Alahmadi TA, Alharbi SA, Sethi G, et al. Attenuation of STAT3 signaling cascade by daidzin can enhance the apoptotic potential of bortezomib against multiple myeloma. Biomolecules. 2019;10:23.

51. Wu K-C, Lin W-Y, Sung Y-T, Wu W-Y, Cheng Y-H, Chen T-S, et al. Glycine tomentella hayata extract and its ingredient daidzin ameliorate cyclophosphamide-induced hemorrhagic cystitis and oxidative stress through the action of antioxidation, anti-fibrosis, and anti-inflammation. Chin J Physiol. 2019:62:188-95.

52. Kazmi Z, Zeeshan S, Khan A, Malik S, Shehzad A, Seo EK, et al. Anti-epileptic activity of daidzin in PTZ-induced mice model by targeting oxidative stress and BDNF/NEGF signaling. Neurotoxicology. 2020;79:150-63.

53. Sun Y, Zhang H, Cheng M, Cao S, Qiao M, Zhang B, et al. New hepatoprotective isoflavone glucosides from Pueraria lobata (Willd.) Ohwi. Nat Prod Res. 2019;33:3485-92.

54. Liu Q, Zuo R, Wang K, Nong F-F, Fu Y-J, Huang S-W, et al. Oroxindin inhibits macrophage NLRP3 inflammasome activation in DSS-induced ulcerative colitis in mice via suppressing TXNIP-dependent NF-KB pathway. Acta Pharmacol Sin. 2020;41:771-81.

55. Deshmukh AB, Datir SS, Bhonde Y, Kelkar N, Samdani P, Tamhane VA. De novo root transcriptome of a medicinally important rare tree Oroxylum indicum for characterization of the flavonoid biosynthesis pathway. Phytochemistry. 2018;156:201-13.

56. Sun Z-G, Zhao T-T, Lu N, Yang Y-A, Zhu H-L. Research progress of glycyrrhizic acid on antiviral activity. Mini Rev Med Chem. 2019;19:826-32.

57. Xu C, Liang C, Sun W, Chen J, Chen X. Glycyrrhizic acid ameliorates myocardial ischemic injury by the regulation of inflammation and oxidative state. Drug Des Devel Ther. 2018;12:1311-9.

58. Huo X, Yang S, Sun X, Meng X, Zhao Y. Protective effect of glycyrrhizic acid on alcoholic liver injury in rats by modulating lipid metabolism. Molecules. 2018;23:1623.

59. Wang Y-M, Du G-Q. Glycyrrhizic acid prevents enteritis through reduction of NF-kB p65 and p38MAPK expression in rat. Mol Med Rep. 2016:13:3639-46.

60. Tan C, Wang A, Liu C, Li Y, Shi Y, Zhou M-S. Puerarin improves vascular insulin resistance and cardiovascular remodeling in salt-sensitive hypertension. Am J Chin Med. 2017;45:1169-84.

61. Lian D, Yuan $H$, Yin $X, W u Y$, He $R$, Huang $Y$, et al. Puerarin inhibits hyperglycemia-induced inter-endothelial junction through suppressing endothelial NIrp3 inflammasome activation via ROS-dependent oxidative pathway. Phytomedicine. 2019;55:310-9.

62. Yang C, Fan X, Fan S. Effects and mechanism of puerarin on the human retinoblastoma cells. J Cell Biochem. 2018;119:4506-13.

63. Zhou $Y-X$, Zhang $H$, Peng C. Puerarin: a review of pharmacological effects. Phytother Res. 2014;28:961-75.

64. Zhao K, Li X, Lin B, Yang D, Zhou Y, Li Z, et al. Oroxyloside inhibits angiogenesis through suppressing internalization of VEGFR2/Flk-1 in endothelial cells. J Cell Physiol. 2018;233:3454-64.
65. Xu Z-F, Sun X-K, Chen G, Han C, Wang F, Zhang Y-D. Oroxyloside inhibits human glioma progression by suppressing proliferation, metastasis and inducing apoptosis related pathways. Biomed Pharmacother. 2018;97:1564-74

66. Liao $Y$, Yang $Y$, Wang $X$, Wei M, Guo Q, Zhao L. Oroxyloside ameliorates acetaminophen-induced hepatotoxicity by inhibiting JNK related apoptosis and necroptosis. J Ethnopharmacol. 2020;258:1117.

67. Liu A, Shen Y, Du Y, Chen J, Pei F, Fu W, et al. Esculin prevents lipopolysaccharide/D-galactosamine-induced acute liver injury in mice. Microb Pathog. 2018;125:418-22.

68. Li W, Wang Y, Wang X, He Z, Liu F, Zhi W, et al. Esculin attenuates endotoxin shock induced by lipopolysaccharide in mouse and NO production in vitro through inhibition of NF-kB activation. Eur J Pharmacol. 2016:791:726-34.

69. Song Y, Wang X, Qin S, Zhou S, Li J, Gao Y. Esculin ameliorates cognitive impairment in experimental diabetic nephropathy and induces antioxidative stress and anti-inflammatory effects via the MAPK pathway. Mol Med Rep. 2018;17:7395-402.

70. Zhang M, Xin X, Lai F, Zhang X, Li X, Wu H. Cellular transport of esculin and its acylated derivatives in Caco-2 cell monolayers and their antioxidant properties in vitro. J Agric Food Chem. 2017;65:7424-32.

71. Chang BY, Jung YS, Yoon C-S, Oh JS, Hong JH, Kim Y-C, et al. Fraxin prevents chemically induced hepatotoxicity by reducing oxidative stress. Molecules. 2017;22:587

72. Ma X, Liu X, Feng J, Zhang D, Huang L, Li D, et al. Fraxin alleviates LPSinduced ARDS by downregulating inflammatory responses and oxidative damages and reducing pulmonary vascular permeability. Inflammation. 2019;42:1901-12.

73. Li Y, Liu X-G, Wang H-Y, Dong $X$, Gao W, Xu X-J, et al. Pharmacokinetic studies of phellodendrine in rat plasma and tissues after intravenous administration using ultra-high performance liquid chromatographytandem mass spectrometry. J Chromatogr B Anal Technol Biomed life Sci. 2016;1029-1030:95-101.

74. Long J, Song J, Zhong L, Liao Y, Liu L, Li X. Palmatine: a review of its pharmacology, toxicity and pharmacokinetics. Biochimie. 2019;162:176-84.

75. Wang K, Feng $X$, Chai L, Cao S, Qiu F. The metabolism of berberine and its contribution to the pharmacological effects. Drug Metab Rev. 2017:49:139-57.

76. Imenshahidi $M$, Hosseinzadeh $H$. Berberis Vulgaris and berberine: an update review. Phytother Res. 2016;30:1745-64.

77. Imenshahidi M, Hosseinzadeh H. Berberine and barberry (Berberis vulgaris): a clinical review. Phytother Res. 2019:33:504-23.

78. Naveed M, Hejazi V, Abbas M, Kamboh AA, Khan GJ, Shumzaid M, et al. Chlorogenic acid (CGA): a pharmacological review and call for further research. Biomed Pharmacother. 2018;97:67-74.

79. Santana-Gálvez J, Cisneros-Zevallos L, Jacobo-Velázquez DA. Chlorogenic acid: recent advances on its dual role as a food additive and a nutraceutical against metabolic syndrome. Molecules. 2017;22:358.

80. Nabavi SF, Tejada S, Setzer WN, Gortzi O, Sureda A, Braidy N, et al. Chlorogenic acid and mental diseases: from chemistry to medicine. Curr Neuropharmacol. 2017:15:471-9.

81. Miao M, Xiang L. Pharmacological action and potential targets of chlorogenic acid. Adv Pharmacol. 2020;87:71-88.

\section{Publisher's Note}

Springer Nature remains neutral with regard to jurisdictional claims in published maps and institutional affiliations. 